\title{
Using Sound and Music in Technology
}

\author{
Gerard N. Foster \\ Purdue University - School of Technology at Kokomo
}

\section{Introduction}

This paper presents work aimed at adding the aspect of sound, and music, to technical demonstrations, presentations and projects. The focus of this work is to explore the mathematics and technology of sound at a basic level, to create a background for later experimentation. To enhance the apparent relevance of technology to everyday concerns, the human voice and musical instruments are employed as sources of sound. It is postulated that by stirring the senses, the imaginations and motivations of students and teachers can be aroused. In its basic form, the vehicle for exploring sound can be the simple transduction of sound with a simple electret microphone procured from Radio Shack and the display of the resulting waveform on an oscilloscope. There are a myriad of more complex applications for sound including all sorts of analog and digital processing. On the path to these explorations, the author and students became involved in the production of musical instruments from ordinary soda straws and ten-foot PVC pipes.

\section{Background}

One of the set of standard projects at the end of our introduction to microprocessors course is the programming of a music box that relies on creating data tables for the generation of various notes of the scale. This project also requires hardware to filter the squarewaves and to amplify and transduce them to sound waves. Knowing what the actual frequencies of the notes are is essential to developing such a project. While essential to this project, this knowledge may never be elucidated by either music teachers or physics teachers. The following discourse is a brief coverage linking the musical and the physical. In discussing the broad aspects of music and especially harmony and discord we are "dealing with subjects which lie partly within the province of science and partly within that of art, and the boundaries between the two provinces is not always perfectly clear." (Jeans, p. 152)

The ambiguities between art and science are immediately evident when we consider the frequencies of the notes in the diatonic and chromatic scales of western music. Musical instruments were played in the ancient world. Frequency relationships can be inferred from a knowledge of the lengths the strings of a lyre or of a pipe from ancient artifacts. The earliest musical knowledge was that of the octave. A note that has a frequency that is twice as great as another note is said to be an octave higher. Successive do's on a scale are an octave apart. If the lower note is called the fundamental, then the higher note is called the second harmonic. Let's assign the note signature $\mathrm{C}$ and $\mathrm{C}^{\prime}$ to these notes in this discussion. The sounding of these two notes together has a pleasurable appeal to humans and they are said to be in consonance or in harmony with one another. The third harmonic is a frequency three times that of the fundamental C. It, too, sounds harmonic when sounded with the other two notes. Jumping one octave lower than the third harmonic, we derive a note in between $\mathrm{C}$ and $\mathrm{C}^{\prime}$ that is our present day $\mathrm{G}$. 
We note that note $\mathrm{G}$ is a set ratio of the note $\mathrm{C}$ as shown in the equation below. The harmonic relationship of these two notes contributes to the consonant sound that we experience when they are played together.

$$
f_{G}=(3 / 2) f_{C}
$$

Continuing to play with this idea of harmonics, we find that there is a note, call it FFF, somewhat less than two octaves lower than $\mathrm{C}$ for which $\mathrm{C}$ is the third harmonic. In this case

$$
f_{C}=3 f_{F F F} \text { and } f_{F F F}=(1 / 3) f_{C} \text {. }
$$

By going up two octaves from FFF we have another note $\mathrm{F}$ as follows

$$
f_{F}=4 f_{F F F}=(4 / 3) f_{C}
$$

This combination of C F G and $\mathrm{C}^{\prime}$ formed the strings on early Greek lyres.

With this play, Pythagoras derived the notes of the scale that had a ratio of frequency from the fundamental as shown below.

$$
\begin{array}{llllllll}
\text { C } & \text { D } & \text { E } & \text { F } & \text { G } & \text { A } & \text { B } & \text { C' }^{\prime} \\
1 & 9 / 8 & 81 / 64 & 4 / 3 & 3 / 2 & 27 / 16 & 243 / 128 & 2
\end{array}
$$

In the key of $\mathrm{C}, \mathrm{G}$ is known as the fifth because it is the fifth harmonic note in the progression. $\mathrm{F}$ is known as the fourth. This scale has "a greater wealth of concords than can be attained from any other selection of eight notes." (Jeans, 168) These intervals were later adjusted to what is known as the Just Scale as shown below.

$\begin{array}{llllllll}\mathrm{C} & \mathrm{D} & \mathrm{E} & \mathrm{F} & \mathrm{G} & \mathrm{A} & \mathrm{B} & \mathrm{C}^{\prime} \\ 1 & 9 / 8 & 5 / 4 & 4 / 3 & 3 / 2 & 5 / 3 & 15 / 8 & 2\end{array}$

The above two scales of seven notes to the eighth "octave" note are called diatonic scales. The frequency intervals E-F and B-C' are approximately half of the intervals of the other tones and, therefore, are called hemitones or half steps. Filling in the remaining half steps, we arrive at a twelve note scale to the thirteenth octave note called the chromatic scale. The new notes are the sharps or flats and are represented on a piano by the black keys.

The scales above, based on considerations of harmonics, are employed by trained singers and violinists with the innate ability to adjust the ratios to the key of the song being sung or/and played. That is true unless a keyboard instrument is a member of the ensemble. Fixed-note instruments like the piano have a problem. The scale ratios above apply to the key (the fundamental note) of the scale. The exact frequencies derived for the key of $\mathrm{C}$ do not apply for other keys. A piano, for instance, could be tuned for the key of $\mathrm{C}$ as soon above. However, the notes thus established would be wrong in a different key. Another system of scale intervals called the Equal Temperament Scale was established to overcome this problem. The solution is to derive each of the tones in the twelve half-step intervals of the chromatic scale by multiplying 
the previous note by the same factor. Since the frequency span of an octave has a ratio of 2 , the factor used to determine successive notes is the twelfth root of 2 which is 1.0595 . The differences in frequencies produced with this Equal Temperament Scale are remarkably similar to those found with the other scaling methods. A piano tuned in this manner can be used to perform "acceptably" in all keys although the harmonic nature of polyphonic music will not be quite realized.

If you wish to calculate the frequencies of musical notes, you can start with the knowledge that the $\mathrm{A}$ above middle $\mathrm{C}$ has been set by international convention to have a frequency of $440 \mathrm{~Hz}$.

Thus, we see that there is a mathematics involved in establishing the notes of the musical scale in western music and that compromises must be made in applying theory to practice. The artist and the author must live with such inexactness just as ultimately students must live with the approximations of any complicated design in technology.

\section{Applications}

Ideas on what comprises good musical composition, what sounds are consonant and what sounds are dissonant are under constant developmental change. It is well beyond the scope of this short paper to explore such aspects. But it is my contention that the processing of voice and music signals can add interest and understanding to the basic concepts that are taught in electrical engineering technology. The applications presented below are some of the ways that the sounds of voice and music can be included in technical laboratories and demonstrations.

\section{Transduction and display of sound}

The function generator is the standard device for the creation of waveforms in the laboratory. Such waveforms can be displayed and measured with an oscilloscope. However, waveforms can also be gathered from physical media and transduced with a microphone and amplifier circuit. The author has successfully used this method on a number of occasions to demonstrate to high school students and teachers the nature of sound, electronics and the physical relationship of vibration to the production of sine waves. The way that the voice appears on an oscilloscope was first demonstrated at a presentation on technology in which the display of the waveform was projected on a large screen via a VCR camera and a projection system while the presenter spoke. Later in the presentation a sine wave was produced with a tuning fork and by whistling. Finally students were asked to demonstrate various waveforms produced by the vowels a e i o and u.

\section{Workshop on sine waves, sound, music and the use of an oscilloscope}

The presentation above lead to two 1 hour workshops for high school students and for teachers. First the participants drew a sine wave by transferring measurements acquired from a circle. Next, a sine wave was generated by a function generator and displayed on an oscilloscope. Then, the output of the function generator was connected to a speaker and the audio acuity of the experimenters was subjectively tested. With this introduction to sine waves and audio signals behind them the experimenters connected a microphone circuit to the oscilloscope and produced sound waves by talking, whistling and playing hand-made musical instruments. For simplicity the microphone circuit used a minimum of readily available parts. The microphone was a simple electret microphone. This microphone was coupled to the input of an LM386 audio amplifier through a 10 microfarad capacitor. Another $10 \mathrm{uF}$ capacitor was used to set the gain of the 
circuit. All components were available from Radio Shack. A tuning fork and a human whistle were shown to produce relatively pure sine waves. The frequency of the tones could be measured on the oscilloscope using cursors. A good whistler could demonstrate by whistling the scale that the frequencies of a low "do" and the next higher "do" in a whistled scale differed by a factor of two. The instructor demonstrated the waves created from the sound of a 10 foot $x 1$ inch PCV pipe. The lowest note of approximately $80 \mathrm{~Hz}$ created a periodic wave that looked like the beginnings of a square wave composed of the fundamental and a few overtone frequencies. By practicing ones embouchure (lip tension and vibration), higher and higher tones could be produced. The higher frequency notes had a tendency to be less square and more sinusoidal. After this demonstration, students were given ordinary plastic soda straws with the instructions to cut two opposing one-half to three-quarter inch wedges at one end of the straw. With one's teeth, the two tabs thus formed can be flattened and made flexible creating a double reed. It takes practice but this straw can be played like a musical instrument. It sounds like a duck call and the resulting waveform is periodic but far from a pure sinusoid. The last exercise of the workshop was to cut the straw to a length given by the Just Scale proportions shown above. Since the effective length of the straw is not the same as the actual length of the straw, this exercise was an inconclusive demonstration of the theory of scales, but the general concept that a shortened pipe length leads to a higher frequency was achieved. The high school students enjoyed the workshop, but the EET student assistants really got a "kick" out of the exercises. They stayed after the others had left and made more instruments including extra finger holes.

\section{Digital play/record circuit}

This last application was really the author's first use of microphones to produce analog signals. A project was introduced in the second level digital fundamentals course. The premise behind the project was to get the students to use a RAM $(8 \mathrm{Kx} 8)$ device that was comparable to one they would see in their introduction to microprocessors course. The previously used RAM was 16x4. This latter device is easy to hand load, but it is not large enough to challenge the students' imaginations as to the applications of a regular memory device in a microcomputer circuit. However, the larger memory device was too large to be conveniently hand loaded. The solution was to use counter circuits to roll through the sequential addresses of the memory. In the record mode, the data was gathered from an A/D converter. The input to the A/D was an electret microphone connected to a zero-span op-amp circuit. In the play mode the output of the A/D was tri-stated and the RAM was flipped to its read mode where the data stored was fed to the input of a DAC and then on to a filter, amplifier and speaker. The sampling rate was approximately $8 \mathrm{kHz}$ and the record time was about 1 second. The fidelity of the output was good enough to identify the voice of the talker. The intent of describing this project is simply to indicate another use for the transduction of voice or music. This project has been used twice with success.

\section{Conclusion}

It is said that students grasp the real, the concrete, much better than the abstract. In that regard technical educators introduce laboratory exercises where waveforms are generated by function generators and are displayed on oscilloscopes. The superposition of sinusoidal waveforms is demonstrated physically to reinforce the mathematical demonstrations of calculated results. Yet the concept of sine and cosine waves is still somewhat abstract. Why not create the waveforms by mechanical means and transduce them with a microphone? That was the intent of the work 
discussed. The result has been that a number of demonstrations and projects have been implemented that illustrate that physical vibrations can be transduced into measurable electric signals and that these signals demonstrate the laws of physics and mathematics such as superposition and the generation of harmonics..

What of the present and future? Currently, we are implementing laboratory projects in our first DSP course that include, for example, the creation of echo and reverberation of sound. The work presented in this paper forms a good foundation for such applications in DSP. Additionally, I can foresee becoming involve in digital interfaces such as MIDI. The field for the application of sound and music is, of course, wide open. And the work described in this paper is but an introduction and an invitation to employ the fascinating phenomena of sound.

\section{Bibliography}

1. Benade, A. H. (1990). Fundamentals of Musical Acoustics (2nd rev. ed.). New York: Dover.

2. Eargle, J. M. (1990). Music, Sound and Technology. New York: VanNostrand Reinhold.

3. Everest, F. A. (1994). The Master Handbook of Acoustics (3rd ed.). NewYork: McGraw-Hill.

4. Fletcher, N. H. \& Rossing, T. D. (1991). The Physics of Musical Instruments. New York: Springer-Verlag.

5. Jeans, J. (1937, 1968). Science \& Music. New York: Dover.

6. Randal, D. M. (1978). Harvard Concise Dictionary of Music. Cambridge, MA: Harvard University.

\section{Biography}

Gerard N. Foster is an associate professor of electrical engineering technology at Purdue University School of Technology at Kokomo, Indiana. He teaches the digital fundamentals and microcontroller sequence of classes. Currently he is teaching a new course on digital signal processing. Professor Foster' interests include development of student projects, microcomputer applications and multimedia. 